\title{
Image Compression using Orthogonal Wavelets Viewed from Peak Signal to Noise Ratio and Computation Time
}

\author{
P.M.K. Prasad \\ Associate Professor \\ Dept of ECE, GMRIT, Rajam, \\ Srikakulam dist, A.P., India.
}

\author{
Prabhakar Telagarapu \\ Assistant Professor \\ Dept of ECE, GMRIT, Rajam, \\ Srikakulam dist, A.P., India.
}

\author{
G. Uma Madhuri \\ Assistant Professor \\ Dept of ECE, GMRIT, Rajam, \\ Srikakulam dist, A.P., India.
}

\begin{abstract}
Uncompressed image data requires considerable storage capacity and transmission bandwidth. Despite rapid progress in mass-storage density, processor speeds, and digital communication system performance, demand for data storage capacity and data transmission bandwidth continues to outstrip the capabilities of available technologies. Images require substantial storage and transmission resources, thus image compression is advantageous to reduce these requirements. Different wavelets will be used to carry out the transform of test image and the results will be analyzed in terms of Peak signal to noise ratio obtained and the computation time taken for decomposition and reconstruction. The orthogonal wavelet used are Daubechies family of Haar (Daubechies 1), Daubechies 2, Daubechies 3, Daubechies 4, Daubechies 5, and Coiflet families, as well as Symlet families.. The wavelet which has the highest PSNR in each family is Haar(db), Coiflet1, andSymlet 2 and less computation time in each family is Haar(db1), symlet3, 4, 6 and coiflet1.
\end{abstract}

\section{Keywords}

Compression, Image, Wavelet, Decomposition level, PSNR.

\section{INTRODUCTION}

Often signals we wish to process are in the time-domain, but in order to process them more easily other information, such as frequency, is required. Mathematical transforms translate the information of signals into different representations. For example, the Fourier transform can be seen. However the Fourier transform cannot provide information on which frequencies occur at specific times in the signal as time and frequency are viewed independently. To solve this problem the Short Term Fourier Transform (STFT)[9] introduced the idea of windows through which different parts of a signal are viewed. For a given window in time the frequencies can be viewed. However Heisenberg's Uncertainty Principle states that as the resolution of the signal improves in the time domain, by zooming on different sections, the frequency resolution gets worse. Ideally, a method of multi-resolution is needed, which allows certain parts of the signal to be resolved well in time, and other parts to be resolved well in frequency. The power and magic of wavelet analysis is exactly this multiresolution.

Images contain large amounts of information that requires much storage space, large transmission bandwidths and long transmission times [1][2[4]. Therefore it is advantageous to compress the image by storing only the essential information needed to reconstruct the image. An image can be thought of as a matrix of pixel (or intensity) values. In order to compress the image, redundancies must be exploited, for example, areas where there is little or no change between pixel values. Therefore images having large areas of uniform color will have large redundancies, and conversely images that have frequent and large changes in color will be less redundant and harder to compress[11][13].

Wavelet analysis can be used to divide the information of an image into approximation and detail sub signals. The approximation sub signal shows the general trend of pixel values, and three detail sub signals show the vertical, horizontal and diagonal details or changes in the image. If these details are very small then they can be set to zero without significantly changing the image. The value below which details are considered small enough to be set to zero is known as the threshold. The greater the number of zeros the greater the compression that can be achieved. The amount of information retained by an image after compression and decomposition is known as the 'energy retained' and this is proportional to the sum of the squares of the pixel values. If the energy retained is $100 \%$ then the compression is known as 'lossless', as the image can be reconstructed exactly. This occurs when the threshold value is set to zero, meaning that the detail has not been changed. If any values are changed then energy will be lost and this is known as 'lossy' compression [10]. Ideally, during compression the number of zeros and the energy retention will be as high as possible. However, as more zeros are obtained more energy is lost, so a balance between the two needs to be found.

\section{WAVELET DECOMPOSITION}

In the decomposition level 1, the image will be divided into 4 sub bands, called LH, LH, HL, and HH [6][8]. The LL sub band is a low-resolution residue that has low frequency components, which are often referred to as the average image. LH provides vertical detailed images. HL provides detailed images in the horizontal direction, The $\mathrm{HH}$ sub band image gives details on the diagonal, the while LL sub band is divided again at the time of decomposition at a higher level i.e. LL subband can be further decomposed into four subbands labeled as LL2, LH2, HL2, and HH2 as shown in Figure.1 The process is repeated in accordance with the desired level. In this paper, we have considered level 2 decomposition.

In the discrete wavelet transform (DWT) [5], [7], there are properties for precise reconstruction. This nature gives a sense that in fact no information is lost after the transformed image is set to its original form. But there are missing information on 
image data compression with wavelet transform that occurs during quantization.

\begin{tabular}{|c|c|c|}
\hline LL2 & HL2 & \multirow{2}{*}{ HL1 } \\
LH2 & HH2 & \\
\hline & & \\
& & \\
LH1 & HH1 \\
\hline
\end{tabular}

Figure 1: Two level Wavelet Decomposition of a 2D image

Information loss due to compression should be minimized to keep the quality of the compression. Compression quality is usually inversely proportional with the memory requirement. A good quality compression is generally achieved in the process of memory consolidation, which generates a small reduction, and vice versa. In other words, there is reciprocal (trade off) between image quality and the size of the compression. The quality of an image is subjective and relative, depending on the observation of the user. One can only say the quality of a good image, but others may disagree. There are two things that can be used as benchmarks of compression quality, the PSNR and compression ratio. PSNR (Peak Signal to Noise Ratio) is one of the parameters that can be used to quantify image quality. PSNR parameter is often used as a benchmark level of similarity between reconstructed images with the original image. A larger PSNR produces better image quality. PSNR equation is illustrated below:

$$
P S N R=20 \log _{10} \frac{255}{\sqrt{M S E}}
$$

where

$$
M S E=\frac{1}{m n} \sum_{y=1}^{m} \sum_{x=1}^{n}\left(I(x, y)-I^{\prime}(x, y)\right)^{2}
$$

$I(x, y)$ is Original Image, $I^{\prime}(x, y)$ is Reconstructed Image

\section{PROPOSED METHOD}

The various sections in this proposed method deals with reading a test image cell.tif with size 159x191 and then multilevel wavelet decomposition is done on it. The coefficients obtained are truncated according to threshold value and compressed image is obtained from this original image is reconstructed and then PSNR values and computation time are compared for different wavelets.

\section{RESULTS \& DISCUSSION}

This paper presents some results of the programs compiled with the help of MATLAB [7]. In addition, this study also presents test results on a wavelet influence towards PSNR, with several types of orthogonal wavelets, namely Haar, Daubechies 2, Daubechies 3, Daubechies 4, Daubechies 5, Coiflet family and Symlet. family.

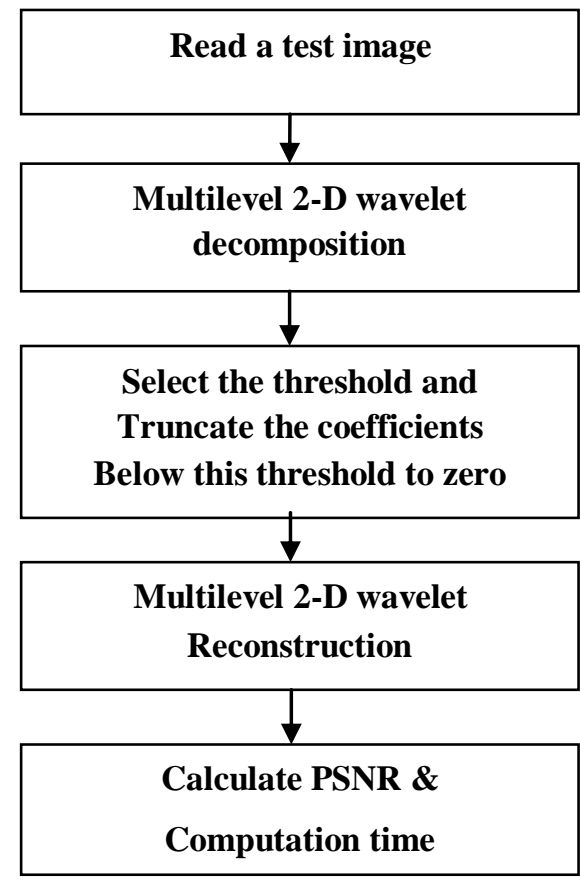

Figure 2: Block Diagram of Proposed Method.

\subsection{The Influence of Wavelet on PSNR}

PSNR is used to quantify the image quality. When the PSNR value is higher, the wavelet functions better, this means that the reconstructed image is likely to be the same as the original image. Table 1 shows the PSNR value with various types of orthogonal wavelets.

Table 1: PSNR values (in $\mathrm{dB}$ ) and Computation Time (in sec) for various Wavelet for cell.tif image

\begin{tabular}{|c|c|c|}
\hline Wavelet & PSNR(dB) & $\begin{array}{c}\text { Computation } \\
\text { Time }(\mathrm{sec})\end{array}$ \\
\hline Haar(db1) & 52.5105 & 0.9375 \\
\hline $\mathrm{db} 2$ & 52.2644 & 0.9688 \\
\hline $\mathrm{db} 3$ & 52.0599 & 1.0313 \\
\hline $\mathrm{db} 4$ & 51.9944 & 0.9531 \\
\hline $\mathrm{db} 5$ & 51.9673 & 1.0625 \\
\hline Coif1 & 52.3504 & 1 \\
\hline Coif2 & 52.0823 & 1.0781 \\
\hline Coif3 & 51.9923 & 1.0781 \\
\hline Coif4 & 51.9839 & 1.1563 \\
\hline Coif5 & 51.9449 & 1.0938 \\
\hline Sym2 & 52.2644 & 1.0469 \\
\hline Sym3 & 52.0599 & 1 \\
\hline Sym4 & 52.0374 & 1 \\
\hline Sym5 & 51.9721 & 1.0469 \\
\hline Sym6 & 51.9669 & 1 \\
\hline Sym10 & 51.9270 & 1.3125 \\
\hline Sym15 & 51.8351 & 1.9063 \\
\hline
\end{tabular}


While the results from testing a wavelet influence on PSNR for cell.tif image can be seen in figure 3 to figure 5 .

\subsubsection{Daubechies family}

Based on table 1 and figure 3, it shows that the wavelet Haar [3] has the highest PSNR value, while the wavelet Daubechies 5 has the lowest.Haar wavelet (db1) has less computation time

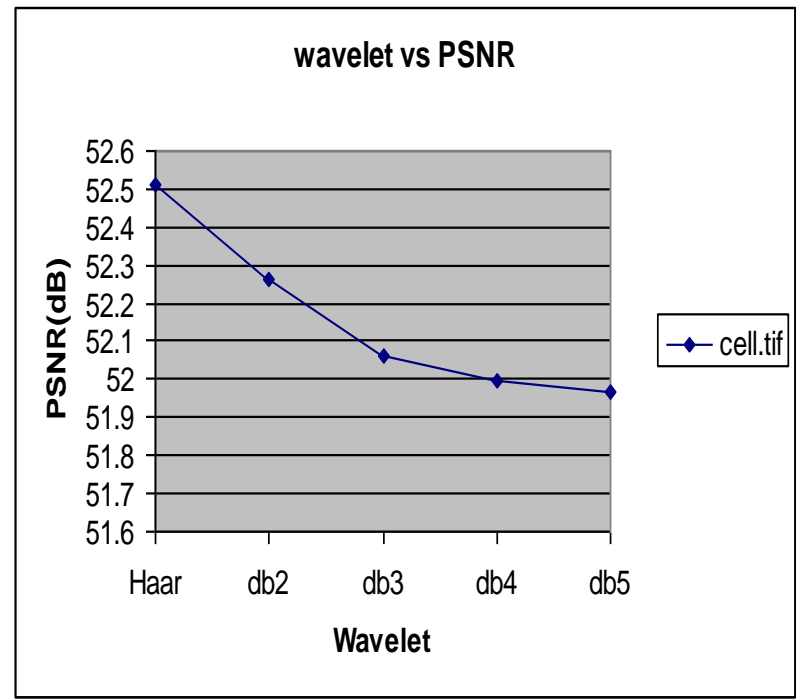

Figure 3. Wavelet versus PSNR (Daubechies family)

\subsubsection{Coiflet Family}

In table 1 and figure 4, it appears that wavelet Coiflet 1 has the highest PSNR, while the wavelet Coiflet 5 has the lowest PSNR. Coif1 wavelet has less computation time

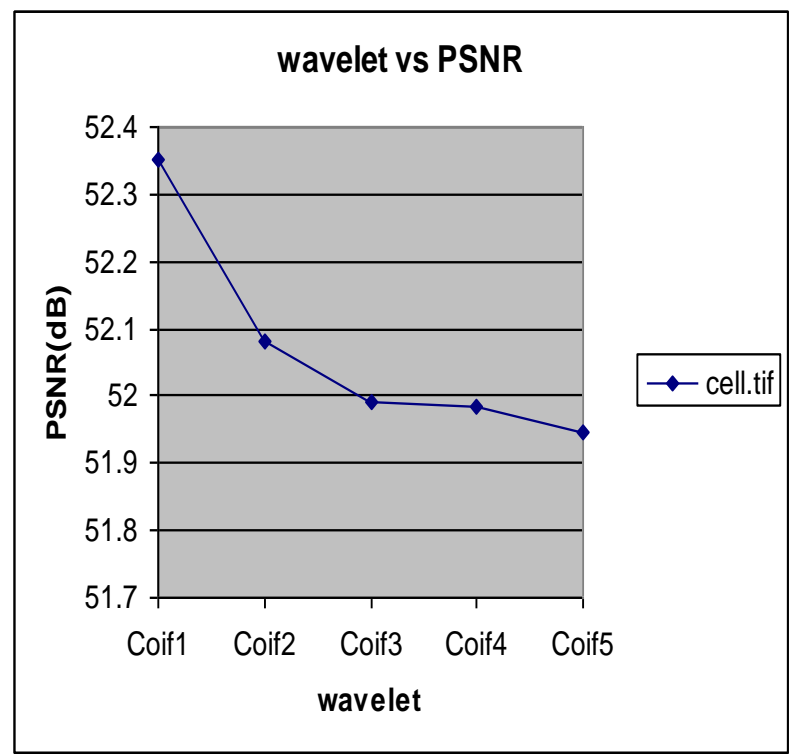

Figure 4: Wavelet versus PSNR (Coiflet Family)

\subsubsection{Symlet Family}

This family of wavelets called symlets, short name for symmetrical wavelets. Based on table 1 and figure 5, it shows that the wavelet symlet2 has the highest PSNR value, while the wavelet symlet 15 has the lowest PSNR.sym3, 4, 6 has less computation time.

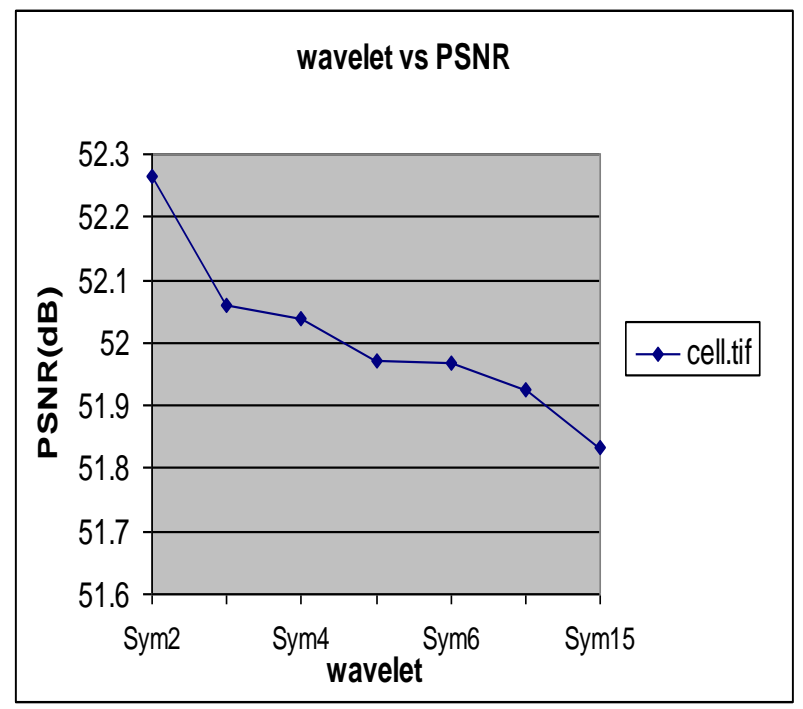

Figure 5.Wavelet versus PSNR (Symlet family)

Table 2 shows the PSNR values and the computation time values of best wavelet from each family obtained by compressing a cell.tif image. Better PSNR and less computation time by Haar (db1) wavelet as shown in the Table 2.

TABLE 2. Best results from each family

\begin{tabular}{|c|c|c|}
\hline $\begin{array}{c}\text { Wavelet } \\
\text { family }\end{array}$ & PSNR(dB) & $\begin{array}{c}\text { Computation time } \\
\text { (sec) }\end{array}$ \\
\hline Haar(db1) & $\begin{array}{c}\text { Haar(db1) } \\
(52.5105)\end{array}$ & $\begin{array}{c}\text { Haar(db1) } \\
(0.9375)\end{array}$ \\
\hline Symlets & $\begin{array}{c}\text { sym2 } \\
(52.2644)\end{array}$ & $\begin{array}{c}\text { sym3,4,6 } \\
(1.000)\end{array}$ \\
\hline Coiflets & $\begin{array}{c}\text { coif1 } \\
(52.3504)\end{array}$ & $\begin{array}{c}\text { coif1 } \\
(1.000)\end{array}$ \\
\hline
\end{tabular}


cell.tif image

\section{Wavelet-Daubeuchies 1}
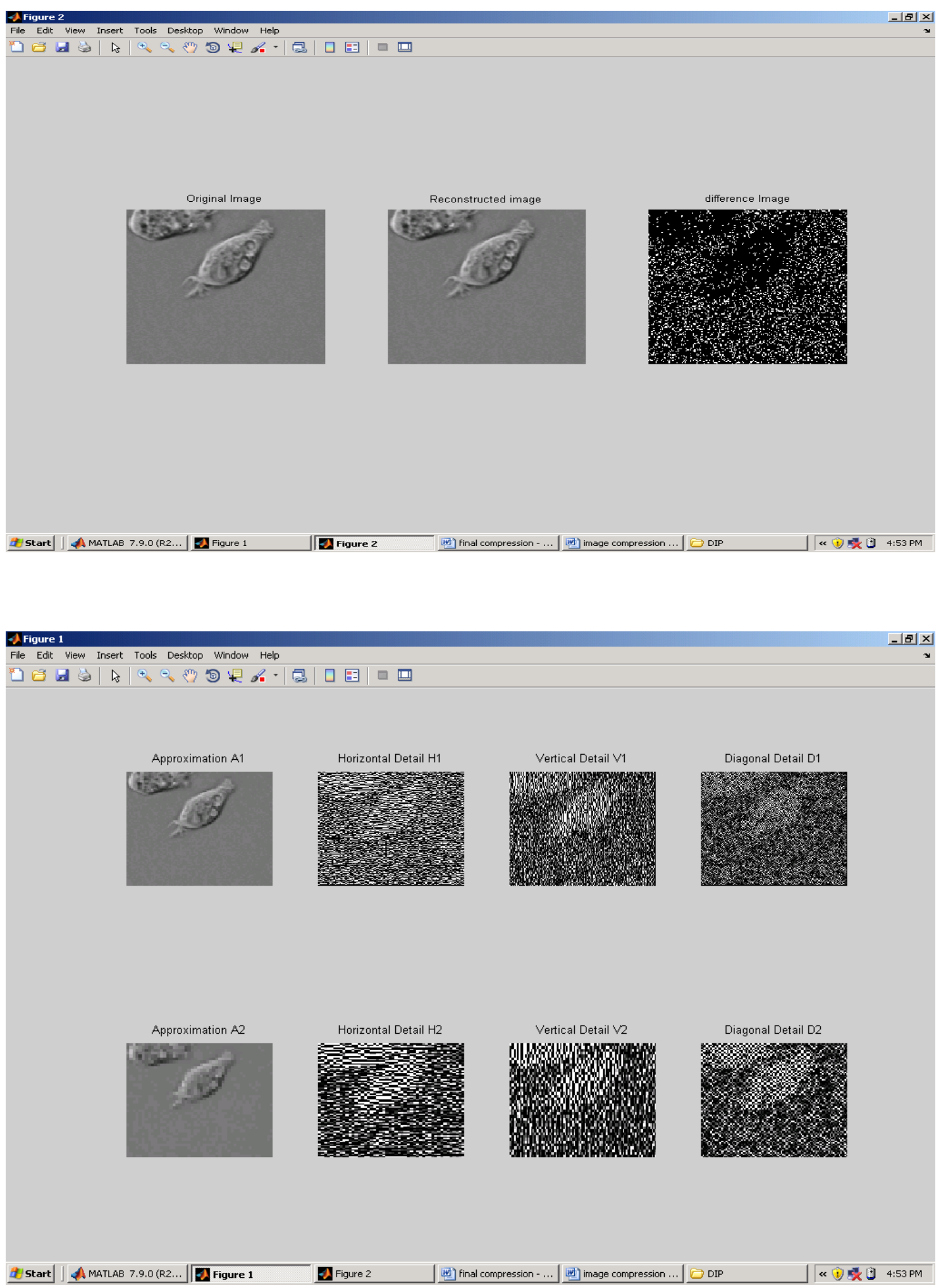

Peak Signal to Noise Ratio: $52.5105 \mathrm{~dB}$

computation Time: $0.9375 \mathrm{sec}$

Figure 6: level 1 and level 2 decomposition levels using db1 

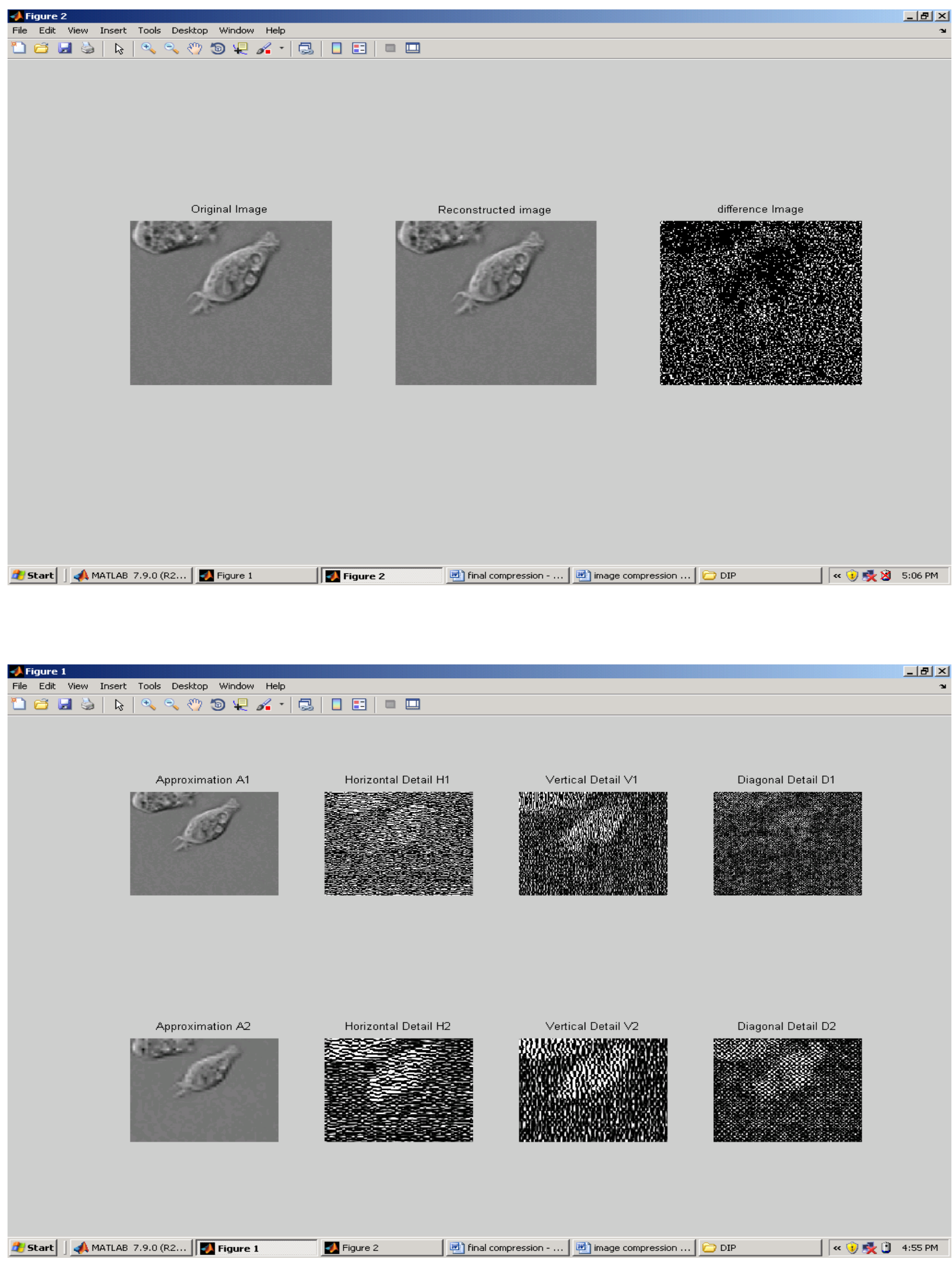

Peak Signal to Noise Ratio: 52.2644 dB

computation Time: $1.0469 \mathrm{sec}$

Figure 7: level 1 and level2 decomposition levels using sym2 

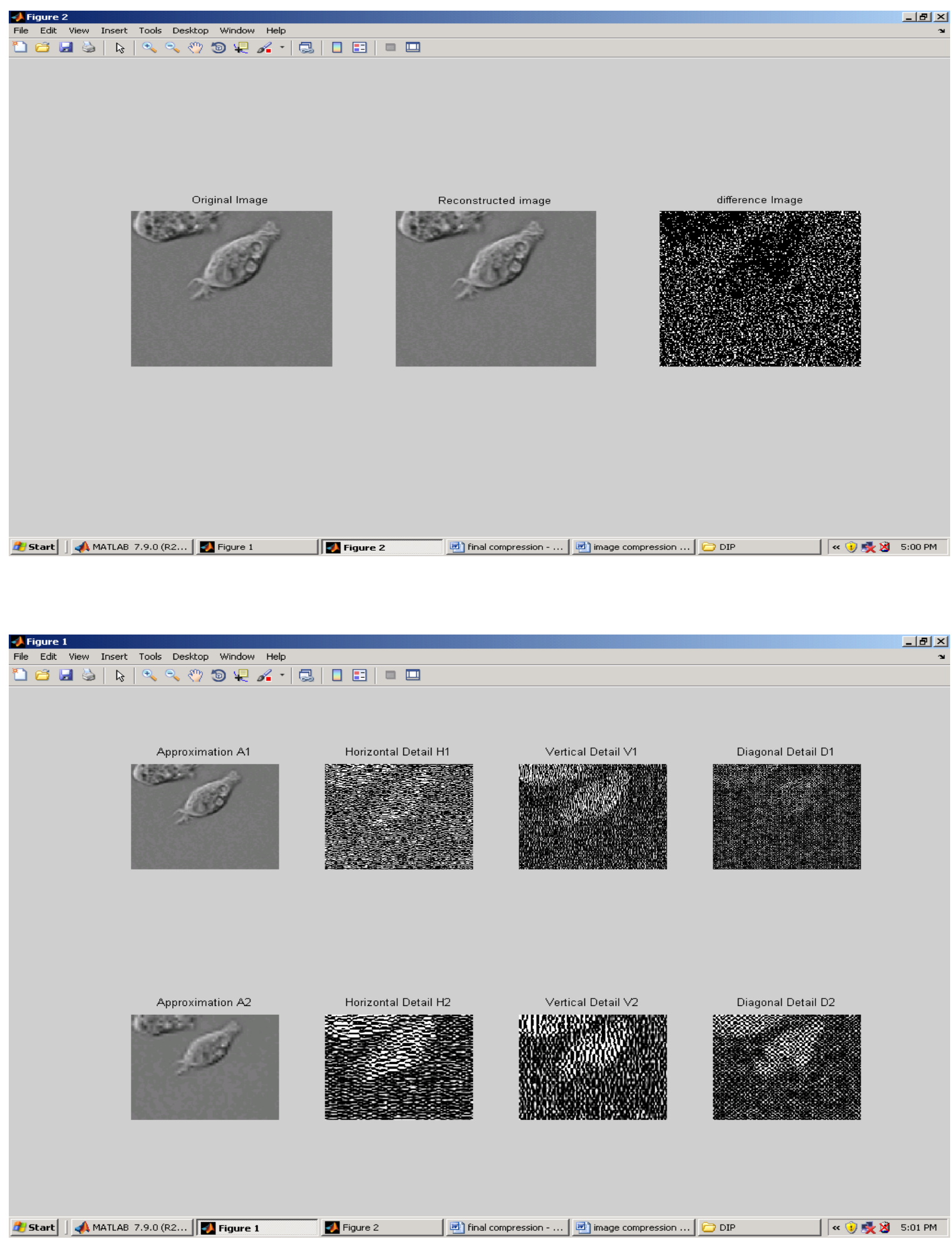

Peak Signal to Noise Ratio: $52.3504 \mathrm{~dB}$

computation Time: $1.0000 \mathrm{sec}$

Figure 8: level 1 and level2 decomposition levels using coiflet1 


\section{CONCLUSION}

In this paper, the obtained results are analyzed and the wavelets which gives high PSNR or which takes less time to compress are tabulated. Wavelet analysis is very powerful and extremely useful for compressing data such as images. Its power comes from its multiresolution. Although other transforms have been used, for example the DCT was used to compress images; wavelet analysis can be seen to be far superior. This is because the wavelet analysis is done on the entire image rather than sections at a time. And also in wavelet analysis both frequency and time domain representations at a time is possible unlike DFT.

Wavelet image compression field has revolutionized image compression field with unbelievable results. Therefore there was a need to exploit the inherent ability of wavelets. The analysis was carried out keeping in mind the fact that if decomposition produces good results it will also give better chances to advanced techniques for further improved results. Generally speaking it is obvious from the results that any wavelet giving good results for decomposition will produce good results for advanced techniques being used for image compression. Based on testing results, it can be concluded that the wavelet Haar, Coiflet 1, and Symlet 2 have the highest PSNR value in every family.

\section{REFERENCES}

[1] R. C. Gonzalez, R. E. woods,"Digital Image processing" second edition, prentice hall of India ltd, 2004.

[2] Raffle C.Gonzalez, Richard E.woods, ,'Digital Image processing using MATLAB", second edition,prentice Hall of India.

[3] K.P.Soman,K.I.Ramachandran, " Insight into wavelets from theory to practice" second edition Prentice Hall of India,2008

[4] Talukder,K.H..,dan Harada K., Haar wavelet Based Approach for Image compression and Quality Assessment of Compressed Image, IAENG International Journal of Applied Mathematics 36:1 IJAM_36_1_9,2007.

[5] Mallat, S, “A Wavelet Tour Of Signal Processing", Academic Press, USA, 1999.
[6] M. Antonini, M. Barland, P. Mathien and I. Daubechies, "Image coding using wavelet transform", IEEE Trans. Image Processing, vol. 1, pp.205-220,April 1992.

[7] S.Grace chang, Bin Yu, Martin Vetterli Adaptive wavelet thresholding for image denoising and compression IEEE transactions on Image processing Vol.9, No.9 September 2000.

[8] S.Jayaraman Esakkirajan, T. veerakumar "Digital Image Processing Tata Mc Graw Hill, publication 2009.

[9] William K. Pratt "Digital image processing", Third Edition, Wiley Student Edition.

[10] Anil.K.Jain "Fundamental of Digital image processing" Fourth Edition, Prentice Hall of India private Limited, 2000.

[11] Rudra pratap “Getting started with MATLAB 7"seventh edition, oxford university press, 2008.

[12] Milan sonka, Vaclav Hlavac, Roger Boyle "Image processing, analysis, and machine vision "second Edition, vikas publishing house, 2001.

\section{ABOUT AUTHOR'S}

P.M.K.Prasad received his M.E in Systems and Signal Processing from Osmania University Hyderabad. He is working as Associate Professor, Department of ECE in GMR Institute of Technology, and Rajam since 2006. His research interests are in Signal Processing, Communication and Image Processing.

Prabhakar Telagarapu received B.E Electronics and Communication Engineering from Andhra University and M.Tech Instrumentation and Control from JNTU Kakinada. $\mathrm{He}$ is working as Assistant Professor Department of ECE in GMR Institute of Technology, Rajam since 2002. His research interests are in Signal Processing, Communication and Image Processing.

G. Uma Madhuri received B.Tech Electronics and Communication Engineering from JNTU Hyderabad. She is working as Assistant Professor Department of ECE in GMR Institute of Technology, Rajam. Her research interests are in Signal Processing, Communication and Image Processing. 